zwischen beiden Wissenschaftsbereichen eine Einigung auf eine Art Forschungsmatrix für das Verständnis kriminologisch relevanter Handlungen geschaffen werden könnte. Ziel dabei müsse es schlicht sein, menschliche Handlungen besser zu verstehen. Doch vor einer derartigen Kooperation von Sozial- und Lebenswissenschaftlern scheint derzeit noch ein steiniger Weg zu liegen.

Dr. Michael Jasch ist wissenschaftlicher Mitarbeiter am Institut für Kriminalwissenschaften der Goethe-Universität Frankfurt am Main. Kontakt:Jasch@jur.uni-frankfurt.de

\section{Fußnoten:}

1 So der suggestive Titel einer Fernsehdokumentation über die Suche nach Kriminalitätsursachen in den Gehirnen verurteilter Gewalttätern, die erstmals 2006 von den Fernsehprogrammen 3sat und Phoenix ausgestrahlt wurde.

2 Wissenschaftliche Veranstalter der Tagung waren die Vorstandsmitglieder der Gesellschaft für interdisziplinäre wissenschaftliche Kriminologie (GiwK).

\section{Literatur:}

Ansermet, Francois; Magistretti, Peter (2005): Die Individualität des Gehirns: Neurobiologie und Psychoanalyse, Frankfurt a.M.

Strasser, Peter (2005): Die Rückkehr der Bio-Wissenschaften in der Kriminologie, in: Pilgram, A.; Prittwitz, C. (Hrsg.): Kriminologie. Akteurin und Kritikerin gesellschaftlicher Entwicklungen (Jahrbuch für Rechts- und Kriminalsoziologie), Baden-Baden.

\title{
Bericht über das Wiener Forum gegen Menschen- handel (Tagung vom 13.-15.02.2008)
}

Ina Hunecke

\begin{abstract}
Menschenhandel ist ein globales Problem. Die Erkenntnis, dass seine Bekämpfung nur durch Zusammenwirken vieler Staaten Erfolg versprechend ist, veranlasste die UN.GIFT ${ }^{1}$ (Global Initiative to fight human trafficking) die erste große UNO-Konferenz mit ca. 1.600 Teilnehmern aus mehr als 128 Staaten vom 13. bis 15. Februar 2008 in Wien einzuberufen.

Gastgeberin war die Nationalratspräsidentin Barbara Prammer. Sie sah die Hauptaufgabe der Tagung darin, nicht nur ein Netzwerk zu knüpfen, sondern einen weiteren Meilenstein im Kampf gegen den Menschenhandel zu setzen. Diesem Wunsch gab sie in ihrer BegrüBungsrede deutlich Ausdruck, als sie sagte: „Ziel müsse es sein, die entsprechenden internationalen Vereinbarungen - die UN-Konvention gegen internationale Verbrechen, das Zusatzprotokoll zur Verhinderung, Verfolgung und Bestrafung von Menschenhandel sowie die diesbezügliche Konvention des Europarates und die Brüsseler Deklaration - zu implementieren und durch konkrete Maßnahmen mit Leben zu füllen.“
\end{abstract}

Der Exekutivdirektor des UN-Büros für Drogen- und Verbrechensbekämpfung Antonio Maria Costa verglich den Menschenhandel mit der Sklaverei und erinnerte an den britischen Unterhaus-Abgeordneten William Wilburforce, der vor 200 Jahren maßgeblich zum Verbot des Sklavenhandels beigetragen hatte. Er rief die Parlamentarier auf, sich daran ein Beispiel zu nehmen und auf nationaler Ebene die notwendigen legislativen Maßnahmen zu treffen und das machtvolle Instrument des UN-Protokolls zur Bekämpfung des Menschenhandels umzusetzen.

Weitere Gastredner waren unter anderen H.E. Suzanne Mubarak, Julia Ormond, Ruslana Lyzhychko, Ricky Martin sowie viele andere Persönlichkeiten aus Politik und Gesellschaft.

Die Themen der Diskussionsforen waren so vielfältig wie die Redner und Besucher ebendieser. Es ging um Prävention, die vielfältigen Gründe für Menschenhandel, die Rolle der Medien, die Frau als Menschenhändler, den Bali Prozess als Model für regionale Kooperation im Kampf gegen Menschenhandel, die Rolle der Jugend, der
Kirchen sowie Glaubengemeinschaften. Es ging um Korruption, um Strategien auch bildungsarme Länder und Schichten zu erreichen sowie immer wieder um die zentrale Frage „Wie kann wer mit wem zusammenarbeiten, um die aktuelle Situation zu verbessern.“

Über die Diskussionsforen hinaus bot die Veranstaltung eine Fülle von Möglichkeiten sich mit dem Thema Menschenhandel sowie Vermeidungs- und Aufklärungsstrategien auseinanderzusetzen.

Eine der kreativen Aktionen startete bereits im Vorfeld des Forums. Die UN.GIFT rief einen weltweiten Comic-Wettbewerb aus, mit dem vor allem Jugendliche erreicht und für das Thema sensibilisiert werden sollten. Die Gewinnercomics des Wettbewerbs waren auf Bannern während des Forums zu sehen und werden in Zukunft von der UN.GIFT genutzt werden, um gerade auch junge Menschen in der ganzen Welt, unabhängig von Sprache und Intellekt zu erreichen und auf das Problem aufmerksam zu machen.

Darüber hinaus wird ein virtuelles Netz erstellt, um weiter miteinander in Kontakt zu bleiben und Aktionen sowie Veranstaltungen planen zu können.

Des Weiteren wurden auch Modellprojekte von Privaten und Unternehmen vorgestellt. Hierfür exemplarisch ist YCI. In diesem Projekt, welches 1995 in Bangkok startete, wird jungen Menschen ein halbes Jahr lang die Möglichkeit gegeben, sich in einem Hotel sowohl theoretisch als auch praktisch ausbilden zu lassen. Die jungen Leute erhalten so eine Perspektive. Wer Arbeit hat, wird sich nicht einem Menschenhändler ausliefern, war eine der gründenden Ideen zu diesem Projekt. Inzwischen gibt es solche „Ausbildungshotels“ auf der ganzen Welt und mehr als 1.600 junge Menschen erhielten so bereits eine Chance auf ein besseres Leben.

Neben den Diskussionsforen setzten sich die Teilnehmer auch cineastisch mit dem Thema auseinander. Gezeigt wurden 19 Filme, darunter auch der auf dem Filmfest in München preisgekrönte Film „Trade“ oder „Blood Diamond“. 
Darüber hinaus gab es während der gesamten Zeit der Konferenz eine Freiluft-Installation am Heldenplatz („,The Journey against sex trafficking“) von der Oscar-Preisträgerin Emma Thompson. In sieben von verschiedenen Künstlern ${ }^{2}$ gestalteten Containern wird der Leidensweg einer jungen Frau beschrieben, die ihr „Glück“ im Ausland suchen will und in die Hände von Menschenhändlern gerät. Die Container tragen Namen wie „Hope“, „Uniform“, „Bedroom“ oder „Customer“. Die Installation war bereits im letzten Jahr mit großem Erfolg in New York und London zu sehen und wird weiter rund um den Golobus reisen.

Abgerundet und beendet wurde die Tagung schließlich durch eine Podiumsdiskussion, in der sich Helga Konrad ${ }^{3}$, Andrew Dismore ${ }^{4}$ sowie Chris Smith ${ }^{5}$ mit der Rolle der Parlamentarier bei der Bekämpfung des Menschenhandels auseinandersetzten.

Mehr Informationen zu dem Wiener Forum gegen Menschenhandel sowie das 19 Seiten starke Draft-summery „The appropriate legal responses to combating trafficking in persons, A handbook for Par- liamentarians" kann man kostenlos unter www.ungift.org oder viennaforum@unov.org erhalten.

Ina Hunecke ist wissenschaftliche Mitarbeiterin an der CAU Kiel

Fußnoten:

1 Die UN-GIFT wurde im März 2007 gemeinsam vom Büro der Vereinten Nationen für Drogen- und Verbrechensbekämpfung (UNODC), der Internationalen Arbeitsorganisation (ILO), der Internationalen Organisation für Migration (IOM), dem Kinderhilfswerk der Vereinten Nationen (UNICEF), dem Hohen Kommissar der Vereinten Nationen für Menschenrechte (UNHCR) und der Organisation für Sicherheit und Zusammenarbeit in Europa (OSZE) gegründet. Innerhalb dieser Initiative bündelt die UNO die Bemühungen mehrerer ihrer Organisationen, die sich bisher parallel mit Teilaspekten dieses Themas befasst haben.

2 z.B. Oscar-Gewinner für Filmdesign: Michael Howells, Oscar-Gewinner für Kostüme: Sandy Powell und Gewinner des Turner-Preises: Anish Kapoor.

3 ehemalige OSZE-Sonderbeauftragte zur Bekämpfung des Menschenhandels

4 Abgeordneter des britischen Unterhauses

5 Mitglied des US-Kongresses

\section{Die Verschärfung des sexuellen Missbrauchs von Jugendlichen und die Kriminalisierung des Besitzes von sog. Jugendpornografie*}

Monika Frommel

Bis 1994 galt im Bereich der alten Bundesrepublik noch eine Jugendschutznorm des $\$ 175$ (Homosexuelle Handlungen). In den neuen Bundesländern galt bis zu diesem Zeitpunkt aufgrund des Einigungsvertrages noch der alte $\$ 149$ StGB-DDR (Einfacher Missbrauch) weiter. Dieser sah ein einheitliches Schutzalter für homo- und heterosexuelle Handlungen vor:

\149. Sexueller Mißbrauch von Jugendlichen. (1) Ein Erwachsener, der einen Jugendlichen zwischen vierzehn und sechzehn Jahren unter Ausnutzung der moralischen Unreife durch Geschenke, Versprechen von Vorteilen oder in ähnlicher Weise dazu mißbraucht, mit ihm Geschlechtsverkehr auszuüben oder geschlechtsverkehrsähnliche Handlungen vorzunehmen, wird mit Freiheitsstrafe bis zu zwei Jahren oder mit Verurteilung auf Bewährung bestraft. (2) Die Strafverfolgung verjährt in zwei Jahren.

1993 einigte man sich auf einen Kompromiss. Die neue einheitlich Strafbestimmung zum Schutz Jugendlicher des $\ 182$ StGB sah ein Schutzalter von 16 und 18 Jahre vor, Täter kann aber de lege lata nur eine Person über 18 Jahre sein. Die geplante Veränderung dieses Kompromisses und die gleichzeitig geplante Verschärfung des absoluten Verbotes der Kinderpornografie auf alle Formen der „Jugendpornografie“ (Definition völlig unklar) verändert die Struktur der bislang geltenden Straftatbestände erheblich und war deshalb bereits mehrfach Gegenstand von Artikeln der NK (NK 4- 2006 und NK 1 2007).

Am 26. August 2006 beschloss das Bundeskabinett - angeblich in Umsetzung europäischer Vorgaben - eine Reihe von Gesetzen zu ändern, da die Schutzaltersgrenzen angeblich in sich widersprüchlich und uneinheitlich seien. Im Vordergrund stand dabei neben einer
Verschärfung des $\ 182$ StGB eine erhebliche Ausweitung und Erweiterung des Verbotes der Kinderpornografie, die nun auf Jugendpornografie (Schutzalter 18 Jahre) erweitert werden soll. Wie eine solche Erweiterung praktisch aussehen soll, ist unklar, da mit dem Wegfall des Bezugs auf den verbotenen sexuellen Missbrauch von Kindern gem. $\$ 176$ StGB sowohl die Tathandlung als auch das Schutzgut völlig unklar wird. Denn das verbot von Pornografie dienst dem Jugendschutz. Jugendliche sollen davon abgehalten werden derartige Darstellungen anzuschauen. Bei der auch für Erwachsene verbotene Gewalt- und Kinderpornografie hat man sich geeinigt, dass derartige Verbote hinzunehmen seien, da schließlich auch sexuelle Kontakte mit Kindern und gewalttätige sexuelle Handlungen verboten seien. Aber das betrachten von Darstellungen Jugendlicher ist Erwachsenen erlaubt. Wieso versucht die Gesetzgebung daher nicht den Datenschutz von Jugendlichen zu verbessern. Denn es sind nicht nur sexuelle Darstellungen im Netz, welche für die weitere Entwicklung dieser dargestellten Jugendlichen schädlich sein können. Bestraft werden Verstöße gegen das informelle Selbstbestimmungsrecht auch de lege lata schon durch das Bundesdatenschutzgesetz. Aber die Umsetzung ist mangelhaft. Zustimmen können Jugendliche nämlich solchen Darstellungen nicht, da sie noch nicht voll geschäftsfähig sind. Man könnte daher Personen unter 18 Jahren schützen. Aber nicht über ein Verbot, das sich gegen erwachsene Betrachter richtet. Von einer solchen Strafgesetzgebung haben die Jugendliche wenig. Der Gesetzesentwurf ist im Dezember 2007 vorerst zurückgezogen worden. Aber wenn die öffentliche Kritik verstummt, ist mit einer Neuauflage zu rechnen. 